\section{Concordance of genetic risk across migraine subgroups: Impact on current and future genetic association studies}

Cephalalgia

20I5, Vol. 35(6) 489-499

(C) International Headache Society 2014 Reprints and permissions: sagepub.co.uk/journalsPermissions.nav DOI: 10.1 |77/0333।024|4547784 cep.sagepub.com

@SAGE

\author{
Dale R Nyholt', for the International Headache Genetics Consortium: \\ Verneri Anttila ${ }^{2,3,4,5}$, Bendik S Winsvold ${ }^{6}$, Tobias Kurth ${ }^{\mathbf{7 , 8}, 9}$, \\ Hreinn Stefansson 10, Mikko Kallela ${ }^{11}$, Rainer Malik'2, \\ Boukje de Vries $^{13}$, Gisela M Terwindt ${ }^{14}$, M Arfan Ikram 15,16,17, \\ Anine H Stam ${ }^{14}$, Lannie Ligthart ${ }^{18,19}$, Tobias Freilinger ${ }^{12,20}$, \\ Michael Alexander ${ }^{21,22}$, Bertram Muller-Myhsok ${ }^{23,24}$, \\ Stefan Schreiber ${ }^{25,26}$, Thomas Meitinger ${ }^{27,28}$, Arpo Aromaa ${ }^{29}$, \\ Johan G Eriksson 29,30,31,32,33, Jaakko Kaprio 34,35,36, \\ Dorret I Boomsma ${ }^{18}$, Cornelia van Duijn ${ }^{15}$, Olli Raitakari ${ }^{37,38}$, \\ Marjo-Riitta Järvelin 39,40,41,42,43, John-Anker Zwart ${ }^{6}$, Lydia Quaye ${ }^{44}$, \\ David P Strachan $^{45}$, Christian Kubisch ${ }^{46,47}$, Michel D Ferrari ${ }^{14}$, \\ Arn $M J$ van den Maagdenberg ${ }^{13,14}$, Martin Dichgans ${ }^{12,24}$, \\ Maija Wessman ${ }^{2,30}$, George Davey Smith ${ }^{48}$, Kari Stefansson ${ }^{10,49}$, \\ Daniel I Chasman' and Aarno Palotie 2,3,5,34,50,5 I
}

\footnotetext{
'Neurogenetics Laboratory, QIMR Berghofer, Australia

${ }^{2}$ Analytical and Translational Genetics Unit, Department of Medicine,

Massachusetts General Hospital, USA

${ }^{3}$ Program in Medical and Population Genetics, Broad Institute of MIT and Harvard, USA

${ }^{4}$ Harvard Medical School, USA

${ }^{5}$ Stanley Center for Psychiatric Research, Broad Institute of Harvard and MIT, USA

${ }^{6}$ FORMI and Department of Neurology, Oslo University Hospital and University of Oslo, Norway

${ }^{7}$ Institut National de la Santé et de la Recherche Médicale (INSERM)

Research Center for Epidemiology and Biostatistics (U897) - Team

Neuroepidemiology, France

${ }^{8}$ University of Bordeaux, France

${ }^{9}$ Department of Medicine, Division of Preventive Medicine, Brigham and

Women's Hospital, Harvard Medical School, USA

${ }^{10} \mathrm{deCODE}$ Genetics, Iceland

"Department of Neurology, Helsinki University Central Hospital, Finland

${ }^{12}$ Institute for Stroke and Dementia Research, Klinikum der Universität München, Ludwig-Maximilians-Universität, Germany

${ }^{13}$ Department of Human Genetics, Leiden University Medical Centre, the Netherlands

${ }^{14}$ Department of Neurology, Leiden University Medical Centre, the Netherlands

${ }^{15}$ Department of Epidemiology, Erasmus University Medical Centre, the Netherlands

${ }^{16}$ Department of Radiology, Erasmus University Medical Centre, the Netherlands

${ }^{17}$ Department of Neurology, Erasmus University Medical Centre, the Netherlands

${ }^{18}$ Department of Biological Psychology, VU University, the Netherlands
}

\footnotetext{
${ }^{19} \mathrm{EMGO}+$ Institute for Health and Care Research, VU University Medical Centre, the Netherlands

${ }^{20}$ Department of Neurology, Klinikum der Universität München, Germany

${ }^{21}$ Department of Genomics, Life \& Brain Center, University of Bonn, Germany

${ }^{22}$ Institute of Human Genetics, University of Bonn, Germany

${ }^{23}$ Max Planck Institute of Psychiatry, Germany

${ }^{24}$ Munich Cluster for Systems Neurology (SyNergy), Germany

${ }^{25}$ Institute of Clinical Molecular Biology, Christian Albrechts University, Germany

${ }^{26}$ Department of Internal Medicine I, Christian Albrechts University, Germany

${ }^{27}$ Institute of Human Genetics, Helmholtz Center Munich, Germany

${ }^{28}$ Institute of Human Genetics, Klinikum Rechts der Isar, Technische

Universität München, Germany

${ }^{29}$ National Institute for Health and Welfare, Finland

${ }^{30}$ Institute of Genetics, Folkhälsan Research Center, Finland

${ }^{31}$ Department of General Practice, Helsinki University Central Hospital, Helsinki, Finland

${ }^{32}$ Vaasa Central Hospital, Finland

${ }^{33}$ Department of General Practice and Primary Health Care, University of Helsinki, Finland

${ }^{34}$ Institute for Molecular Medicine Finland (FIMM), University of Helsinki, Finland

${ }^{35}$ Department of Public Health, Hjelt Institute, University of Helsinki, Finland
}

Corresponding author:

Dale R Nyholt, Neurogenetics Laboratory, QIMR Berghofer, Locked Bag, 2000 Royal Brisbane Hospital, QLD 4029, Australia.

Email: Dale.Nyholt@qimrberghofer.edu.au 


\begin{abstract}
Background: There has been intensive debate whether migraine with aura (MA) and migraine without aura (MO) should be considered distinct subtypes or part of the same disease spectrum. There is also discussion to what extent migraine cases collected in specialised headache clinics differ from cases from population cohorts, and how female cases differ from male cases with respect to their migraine. To assess the genetic overlap between these migraine subgroups, we examined genome-wide association (GWA) results from analysis of 23,285 migraine cases and 95,425 populationmatched controls.

Methods: Detailed heterogeneity analysis of single-nucleotide polymorphism (SNP) effects (odds ratios) between migraine subgroups was performed for the 12 independent SNP loci significantly associated $\left(p<5 \times 10^{-8}\right.$; thus surpassing the threshold for genome-wide significance) with migraine susceptibility. Overall genetic overlap was assessed using SNP effect concordance analysis (SECA) at over 23,000 independent SNPs.

Results: Significant heterogeneity of SNP effects $\left(p_{\text {het }}<1.4 \times 10^{-3}\right)$ was observed between the MA and MO subgroups (for SNP rs9349379), and between the clinic- and population-based subgroups (for SNPs rs 10915437, rs6790925 and rs647824I). However, for all I2 SNPs the risk-increasing allele was the same, and SECA found the majority of genomewide SNP effects to be in the same direction across the subgroups.

Conclusions: Any differences in common genetic risk across these subgroups are outweighed by the similarities. Metaanalysis of additional migraine GWA datasets, regardless of their major subgroup composition, will identify new susceptibility loci for migraine.
\end{abstract}

\title{
Keywords
}

Migraine, subgroups, genome-wide, association, genetic, heterogeneity

Date received: 18 July 20I4; revised: 22 July 20I4; accepted: 23 July 2014

\section{Introduction}

There has been extensive discussion in the migraine field concerning whether migraine with aura (MA) and migraine without aura (MO) are distinct subtypes

\footnotetext{
${ }^{36}$ Department of Mental Health and Alcohol Research, National Institute for Health and Welfare, Finland

${ }^{37}$ Research Centre of Applied and Preventive Cardiovascular Medicine, University of Turku, Turku University Hospital, Finland

${ }^{38}$ Department of Clinical Physiology and Nuclear Medicine, Turku University Hospital, Finland

${ }^{39}$ Institute of Health Sciences, University of Oulu, Finland

${ }^{40}$ Unit of Primary Care, Oulu University Hospital, Finland

${ }^{41}$ Department of Children, Young People and Families, National Institute for Health and Welfare, Finland

${ }^{42}$ Department of Epidemiology and Biostatistics, School of Public Health, MRC-Health Protection Agency (HPA) Centre for Environment and

Health, Faculty of Medicine, Imperial College, UK

${ }^{43}$ Biocenter Oulu, University of Oulu, Finland

${ }^{44}$ Department of Twin Research and Genetic Epidemiology, King's College London, UK

${ }^{45}$ Division of Population Health Sciences and Education, St George's, University of London, UK

${ }^{46}$ Institute of Human Genetics, University Medical Center HamburgEppendorf, Germany

${ }^{47}$ Institute of Human Genetics, University of Ulm, Germany

${ }^{48}$ Medical Research Council Integrative Epidemiology Unit (IEU) at the University of Bristol, School of Social and Community Medicine, UK

${ }^{49}$ School of Medicine, University of Iceland, Iceland

${ }^{50}$ Psychiatric \& Neurodevelopmental Genetics Unit, Department of Psychiatry, Massachusetts General Hospital, USA

${ }^{51}$ Department of Neurology, Massachusetts General Hospital, USA
}

or part of the same disease spectrum (1-4). Similar discussions concern how migraine cases ascertained from headache clinics compare to cases drawn from the general population, and how female migraine cases compare to male cases. In the current genomic age, a particularly important question is whether these major migraine subgroups have predominantly similar or distinct genetic aetiologies.

Results from the latest International Headache Genetics Consortium (IHGC) genome-wide association (GWA) meta-analysis of 23,285 migraine cases and 95,425 controls of European ancestry identified 142 single-nucleotide polymorphisms (SNPs) at 12 loci significantly associated $\left(p<5 \times 10^{-8}\right.$; thus surpassing the threshold for genome-wide significance) with migraine susceptibility. In addition, 1168 SNPs at 134 loci showed suggestive association $\left(p<1 \times 10^{-5}\right)$ with migraine (5). In the original study, SNP effect (odds ratio (OR)) heterogeneity (Cochran's Q) and twotailed binomial tests were utilised to assess whether the effect size of the 146 index (i.e. most strongly associated independent) SNPs significantly differed between the MA and MO, clinic- and population-based, and female and male subgroups. Although minimal SNP effect heterogeneity was observed at the 146 loci, subgroup comparisons for the genome-wide significant loci indicated the effect sizes were predominantly larger in the MO cases compared to MA cases, larger in the clinic-based compared to population-based cases, and 
remarkably similar in female and male cases. These results held for the clinic- versus population-based and female versus male case comparisons for the 146 index SNPs. Analysis of the 146 index SNPs with concordant effect directions in the MA and MO subgroups showed that only $49 \%$ had a larger effect size in $\mathrm{MO}$ cases compared to MA cases, which does not differ significantly from that expected by chance (two-tailed binomial test $p=0.93$ ). However, given these 146 index SNPs were identified via a fixed-effect meta-analysis (which assumes that the true effect is the same in all studies), it is not surprising minimal heterogeneity was observed at these loci.

To more thoroughly assess the overall genetic overlap between these major migraine subgroups, we extend the analyses to SNP effects covering the entire autosome utilising our recently developed web-based application (SECA) to perform SNP effect concordance analysis (6) using GWA summary results from the $\overline{\mathrm{IHGC}}$ (5). Also, for the 12 genome-wide significant loci, we provide detailed results from SNP effect heterogeneity analyses between the MA and MO, clinicand population-based, and female and male subgroups.

\section{Methods}

\section{Ethics statement}

For all study cohorts, participation was based on informed consent. Each study was approved by local research ethics boards in the country where the study cohort was collected. See the original publication of the IHGC GWA meta-analysis for full details of ethics and consent procedures in each study cohort (5).

\section{Cohorts and sample collection}

The IHGC GWA meta-analysis utilised SNP marker data from 23,285 cases with migraine and 95,425 controls of European ancestry from 29 studies, including five clinic-based studies, mainly compared to population-matched control samples with unknown migraine status, as well as 13 population-based cohorts (5). To facilitate comparison of relative subgroup size and power, the effective sample size $\left(N_{\text {eff }}\right)=4 /\left(1 / N_{\text {cases }}+1 /\right.$ $N_{\text {controls }}$ ) was calculated (7). For a subset of the cohorts sufficiently detailed phenotype information was available to allow sub-classification into either of the two migraine subtypes, migraine with aura (MA: 5118 cases versus 74,239 controls, $N_{\text {eff }}=19,152$ ) or migraine without aura (MO: 7107 cases versus 69,427 controls, $\left.N_{\text {eff }}=25,788\right)$. The GWA data were also grouped into clinic-based (5175 cases versus 13,972 controls, $\left.N_{\text {eff }}=15,105\right)$ versus population-based $(18,110$ cases versus 81,453 controls, $\left.N_{\text {eff }}=59,264\right)$, and females-only
(20,202 cases versus 63,593 controls, $\left.N_{\text {eff }}=61,326\right)$ versus males-only (3083 cases versus 31,832 controls, $N_{\text {eff }}=11,243$ ). For more detailed descriptions of the cohorts, please see the original report (5).

\section{GWA results}

Genome-wide SNP genotyping was performed independently in each cohort with the use of various standard genotyping technologies, and imputed for each study with reference to HapMap release 21 or 22 CEU phased genotypes (8). Each study contributed summary statistic data from an association analysis performed using a frequentist additive model based on estimated SNP allelic dosages, adjusting for gender. SNPs were filtered on a per-study level based on inclusion criteria of minor allele frequency (MAF) $>0.1 \%$ and imputation quality measures of $I_{\mathrm{A}}>0.6$ (IMPUTE 2) (9) or $r^{2}>0.3(\mathrm{MaCH})$ (10). In the meta-analysis, combined association data for 3,015,196 unique imputed and genotyped autosomal SNPs were analysed in a fixed-effect model using GWAMA (11). Inconsistent SNP results were filtered out if they produced a very large heterogeneity coefficient $\left(I^{2}>75 \%\right)$ or were present in less than five studies. Less than $2 \%$ of SNPs produced $I^{2}>75 \%$. There was little evidence of population stratification at the study level (genomic inflation factors, $\lambda \leq 1.1$ ), while moderate, but expected inflation was observed at the meta-analysis level $(\lambda=1.13)$ (12). For a complete description, see the original publication of the 2013 IHGC migraine GWA meta-analysis (5).

\section{SNP effect heterogeneity analysis}

Cochran's $\mathrm{Q}$ heterogeneity statistics and $p$ values $\left(P_{\text {het }}\right)$ were estimated via fixed-effect meta-analysis of the OR and $95 \%$ confidence interval $(95 \% \mathrm{CI})$ for each SNP using GWAMA (11). Heterogeneity $p$ values $\left(p_{\text {het }}\right)$ were estimated within and between the MA and MO, clinicand population-based, and female and male subgroups. When the number of studies combined is small $(<10)$ (13), the test has low power to detect heterogeneity if present, while if the number of studies is large, the test is likely to indicate heterogeneity, even if the absolute magnitude of the variability is unimportant (14). Considering the large number and size of the individual migraine GWA studies, we used $p_{\text {het }} \leq 0.05$ to indicate nominal evidence for SNP effect heterogeneity.

\section{SECA approach}

To assess the overall genetic overlap between the MA and MO, clinic- and population-based, and female and male subgroups, SECA (6) compared three sets of two 
(i.e. (MA, MO), (clinic-based, population-based) and (females, males)) GWA summary results, each containing five essential columns: i) reference SNP cluster 'rs' identification (ID) (SNP), ii) effect allele (EA), iii) noneffect allele (NEA), iv) $p$ value from association test $(p)$, and v) OR for the EA relative to the NEA. To ensure consistency across the subgroup comparisons, only SNPs passing quality control (QC) in all subgroups were utilised $(n=1,680,313)$.

SECA first aligned the SNP effects across the two GWA study summary results (dataset1, dataset 2 ) to the same EA, and extracted a subset of independent SNPs via ' $p$ value informed' linkage disequilibrium (LD) clumping using the PLINK program (15). The approach iterates from the first to last SNP on each chromosome sorted from smallest to largest $p$ value in dataset $1\left(P_{1}\right)$ that has not already been clumped (denoting this as the index SNP) and formed clumps of all other SNPs that are within $1 \mathrm{Mb}$ and in LD $\left(r^{2}>0.1\right)$ with the index SNP based on HapMap2 genotype data. A second round of LD clumping was performed to clump any of the round 1 index SNPs within $10 \mathrm{Mb}$ of each other to account for long-range LD $\left(r^{2}>0.1\right)$. The approach identifies the subset of independent SNPs with the most significant association $p$ values $\left(P_{1}\right)$ in dataset 1 .

Restricting to SNPs associated with $p_{1} \leq(0.01,0.05$, $0.1,0.2,0.3,0.4,0.5,0.6,0.7,0.8,0.9,1.0)$ in dataset 1 , SECA performed Fisher's exact statistical tests using the $\mathrm{R}$ statistical package (16) to determine whether there is an excess of SNPs where the effect directions (OR) are concordant across dataset 1 and dataset 2 for the subset of SNPs associated with $p_{2} \leq(0.01,0.05,0.1,0.2,0.3$, $0.4,0.5,0.6,0.7,0.8,0.9,1.0)$ in dataset 2 . For each of the 144 SNP subsets (generated using these $12 \times 12 p$ value threshold combinations), Fisher's exact statistical tests of $\mathrm{SNP}$ effects in dataset 1 (i.e. $\left.\mathrm{OR}_{1}\right)$ and dataset $2\left(\mathrm{OR}_{2}\right)$ were performed on $2 \times 2$ tables containing the number of SNPs with $\mathrm{OR}_{1}<1$ and $\mathrm{OR}_{2}<1, \mathrm{OR}_{1} \geq 1$ and $\mathrm{OR}_{2}<1, \quad \mathrm{OR}_{1}<1$ and $\mathrm{OR}_{2} \geq 1$, and $\mathrm{OR}_{1} \geq 1$ and $\mathrm{OR}_{2} \geq 1$. The proportion of SNP subsets with concordant (Fisher's test $\left.\left(\mathrm{OR}_{\mathrm{FT}}\right) \geq 1\right)$ and discordant $\left(\mathrm{OR}_{\mathrm{FT}}<1\right)$ SNP effects, together with an empirical $p$ value adjusted for testing all 144 subsets $\left(p_{\text {FTsig-per- }}\right.$ muted), was calculated via permutation (1000 replicates) for the observed number of subsets $\left(n_{\mathrm{FTsig}}\right)$ with nominally significant concordance $\left(\mathrm{OR}_{\mathrm{FT}} \geq 1\right.$ and $\left.p_{\mathrm{FT}} \leq 0.05\right)$. A permuted $p$ value was also estimated for the single most significant concordant test $\left(p_{\text {FTmin-permuted }}\right)$. The permutation procedure first created uncorrelated datasets by randomly shuffling the observed SNP effect $\left(\mathrm{OR}_{1}\right)$ and corresponding $p$ value $\left(p_{1}\right)$ between SNPs in dataset1, and then repeats the analysis of the 144 SNP subsets. The SECA web-based application performs numerous other analyses - including pleiotropy tests agnostic to SNP effect direction, Q-Q and true discovery rate (TDR) plots, and 'pleiotropy-informed' conditional false discovery rate (FDR) results for dataset $2 p$ values conditioned on dataset $1 p$ values (6) - but here we report results only from the primary SNP effect concordance tests.

\section{Comparison of SNP effect magnitude}

An effectively random subset of independent SNPs were identified using an LD clumping approach analogous to above, except $p$ values $\left(p_{1}\right)$ were dummy-coded as 0.5 during the clumping rounds. SNP effect comparisons were performed for SNPs with concordant effects in the MA and MO, clinic- and population-based, and female and male subgroup pairs. Exact binomial statistical tests using the $\mathrm{R}$ statistical package (16) were performed to determine whether there was an excess proportion of SNPs with effect magnitudes larger in one subgroup (than the null proportion of 0.5 ) for the random subset of independent SNPs.

The global distribution of effect heterogeneity for the random subset of independent SNPs was also examined by generating Q-Q plots for Cochran's Q $p$ values ( $\left.p_{\text {het }}\right)$. $\mathrm{Q}-\mathrm{Q}$ plots were constructed by ranking the $p$ values from smallest to largest (the 'order statistics') and plotting them against their expected values under the null hypothesis of no association (sampled from the known chi-squared distribution). Deviations above the line of equality (drawn in white) indicate a preponderance of smaller $p_{\text {het }}$ values. To aid interpretation we have also calculated $95 \%$ confidence envelopes (shaded grey in all Q-Q plots). These are formed by calculating, for each order statistic, the 2.5th and 97.5th centiles of the distribution of the order statistic under random sampling and the null hypothesis.

\section{Results}

Associations results for index SNPs at the 12 genomewide significant loci reported in Anttila et al. (2013) (5) are presented in Table 1. In addition to detailed results for the group or subgroup producing the most significant association $p$ value ( $p_{\text {assoc }}$ ), additional groups or subgroups producing genome-wide significant $p_{\text {assoc }}$ values are listed. As for Table 1 in the original study (5), Table 1 lists the $p$ value for Cochran's Q heterogeneity tests $\left(p_{\text {het }}\right)$ for the group or subgroup producing the most significant $P_{\text {assoc }}$ value. To assess overall heterogeneity for the 12 index SNPs across all GWA cohorts, the association results from meta-analysis of all migraine cases are provided in Table 2. Compared to Table 1, which lists one SNP (rs2274316) with nominal heterogeneity $\left(p_{\text {het }}=1.6 \times 10^{-2}\right)$, Table 2 lists three SNPs (rs2274316, rs6790925, rs6478241) with nominal 
Table I. SNP associations results for the 12 genome-wide significant loci reported in Anttila et al. (20I3) (5).

\begin{tabular}{|c|c|c|c|c|c|c|c|c|c|}
\hline \multirow[b]{2}{*}{ SNP } & \multirow[b]{2}{*}{ Chr. } & \multirow[b]{2}{*}{$\begin{array}{l}\text { Position } \\
\text { (bp) }\end{array}$} & \multirow[b]{2}{*}{ RA } & \multirow[b]{2}{*}{ OA } & \multicolumn{3}{|l|}{ Lowest Passoc } & \multirow{2}{*}{$\begin{array}{l}\text { Group or } \\
\text { subgroup with } \\
\text { the lowest } p_{\text {assoc }}\end{array}$} & \multirow{2}{*}{$\begin{array}{l}\text { Additional group } \\
\text { or subgroup with } \\
\text { significant } P_{\text {assoc }}\end{array}$} \\
\hline & & & & & OR $(95 \% \mathrm{Cl})$ & Passoc & Phet & & \\
\hline rs2651899 & I & 3083712 & C & $\mathrm{T}$ & $1.09(1.07-1.11)$ & $4.3 \times 10^{-14}$ & 0.23 & All & $\begin{array}{l}\text { Females, } \\
\text { population-based }\end{array}$ \\
\hline rs10915437 & I & 4183006 & A & G & $1.16(1.10-1.23)$ & $2.8 \times 10^{-8}$ & 0.11 & Clinic-based & \\
\hline rs I 2134493 & I & I I 5677946 & A & C & $1.14(1.10-1.18)$ & $4.8 \times 10^{-14}$ & 0.45 & All & $\begin{array}{l}\text { Females, } \\
\text { population-based }\end{array}$ \\
\hline rs2274316 & I & 156446242 & C & A & $1.07(1.05-1.10)$ & $1.4 \times 10^{-8}$ & $1.6 \times 10^{-2}$ & All & \\
\hline rs7577262 & 2 & 234818869 & G & $A$ & $1.15(1.11-1.19)$ & $3.3 \times 10^{-13}$ & $9.5 \times 10^{-2}$ & All & $\begin{array}{l}\text { Females, } \\
\text { MO, clinic-based }\end{array}$ \\
\hline rs6790925 & 3 & 30480085 & $\mathrm{~T}$ & C & $1.15(1.10-1.21)$ & $2.2 \times 10^{-8}$ & 0.78 & Clinic-based & \\
\hline rs9349379 & 6 & 12903957 & A & G & $1.17(1.11-1.23)$ & $2.8 \times 10^{-10}$ & 0.44 & MO & All \\
\hline rs|320832I & 6 & 96860354 & A & G & $1.18(1.13-1.24)$ & $2.1 \times 10^{-12}$ & 0.56 & MO & All, females \\
\hline rs4379368 & 7 & 40466200 & $\mathrm{~T}$ & C & I.II (1.08-I.I5) & $1.1 \times 10^{-9}$ & 0.44 & All & Females \\
\hline rsI050486I & 8 & 89547932 & C & $\mathrm{T}$ & $1.17(1.11-1.23)$ & $1.2 \times 10^{-8}$ & 0.76 & MO & \\
\hline rs647824I & 9 & 119252629 & A & G & $1.16(1.11-1.22)$ & $1.0 \times 10^{-9}$ & 0.65 & Clinic-based & \\
\hline rsIII72II3 & 12 & 57527283 & $\mathrm{~T}$ & C & I.II (I.08-I.I3) & $3.9 \times 10^{-19}$ & 0.20 & All & $\begin{array}{l}\text { Females, population- } \\
\text { based, MO }\end{array}$ \\
\hline
\end{tabular}

SNP: single nucleotide polymorphism; rsID: reference SNP cluster identification; Chr:: chromosome; Position (bp): genomic position (base pairs) relative to hg 19; RA: risk allele; OA: other allele; OR $(95 \% \mathrm{Cl})$ : odds ratio and $95 \%$ confidence interval for the RA; $p_{\text {assoc: }} p$ value for allelic association; $p_{\text {het }}: p$ value for Cochran's $Q$ heterogeneity test; MO: migraine without aura.

Table 2. SNP association results in all 2013 IHGC samples.

\begin{tabular}{|c|c|c|c|c|c|}
\hline SNP & RA & OA & OR $(95 \% \mathrm{Cl})$ & Passoc & $P_{\text {het }}$ \\
\hline rs2651899 & C & $\mathrm{T}$ & $1.09(1.07-1.11)$ & $4.3 \times 10^{-14}$ & 0.24 \\
\hline rs10915437 & $A$ & G & 1.05 (1.02-1.07) & $6.1 \times 10^{-4}$ & $8.1 \times 10^{-5}$ \\
\hline rs 12134493 & $A$ & C & $1.14(1.10-1.18)$ & $4.8 \times 10^{-14}$ & 0.45 \\
\hline rs2274316 & C & $A$ & $1.07(1.05-1.10)$ & $1.4 \times 10^{-8}$ & $1.6 \times 10^{-2}$ \\
\hline rs7577262 & G & $A$ & $1.15(1.11-1.19)$ & $3.3 \times 10^{-13}$ & $9.5 \times 10^{-2}$ \\
\hline rs6790925 & $\mathrm{T}$ & C & $1.05(1.02-1.07)$ & $8.7 \times 10^{-5}$ & $1.2 \times 10^{-2}$ \\
\hline rs9349379 & $A$ & G & $1.07(1.05-1.10)$ & $4.6 \times 10^{-8}$ & $8.0 \times 10^{-2}$ \\
\hline rs|320832| & $A$ & G & $1.10(1.07-1.12)$ & $1.4 \times 10^{-11}$ & 0.15 \\
\hline rs4379368 & $\mathrm{T}$ & C & $1.11(1.08-1.15)$ & $1.1 \times 10^{-9}$ & 0.44 \\
\hline rs $1050486 \mid$ & C & $\mathrm{T}$ & $1.04(1.01-1.08)$ & $3.5 \times 10^{-3}$ & 0.15 \\
\hline rs647824I & $A$ & G & $1.06(1.04-1.09)$ & $4.4 \times 10^{-7}$ & $2.6 \times 10^{-2}$ \\
\hline rs 11172113 & $\mathrm{~T}$ & C & $1.11(1.08-1.13)$ & $3.9 \times 10^{-19}$ & 0.20 \\
\hline
\end{tabular}

SNP: single nucleotide polymorphism; rsID: reference SNP cluster identification; IHGC: International Headache Genetics Consortium; RA: risk allele; OA: other allele; OR $(95 \% \mathrm{Cl})$ : odds ratio and $95 \%$ confidence interval for the RA; $p_{\text {assoc: }} p$ value for allelic association; phet: $p$ value for Cochran's $Q$ heterogeneity test.

heterogeneity $\left(1.2 \times 10^{-2} \leq p_{\text {het }} \leq 2.6 \times 10^{-2}\right)$ and one SNP (rs10915437) with highly significant heterogeneity $\left(p_{\text {het }}=8.1 \times 10^{-5}\right)$.

Comparing the 12 index SNP effects between the MA and MO subgroups (Table 3), we found four
SNPs (rs10915437, rs9349379, rs13208321 and rs10504861) with heterogeneous effect size ( $p_{\text {het }}=$ $4.4 \times 10^{-3}, \quad 3.2 \times 10^{-4}, 4.9 \times 10^{-2}$ and $4.5 \times 10^{-3}$, respectively). Apart from the effect for rs10915437, which was exclusively found in the MA subgroup, the 


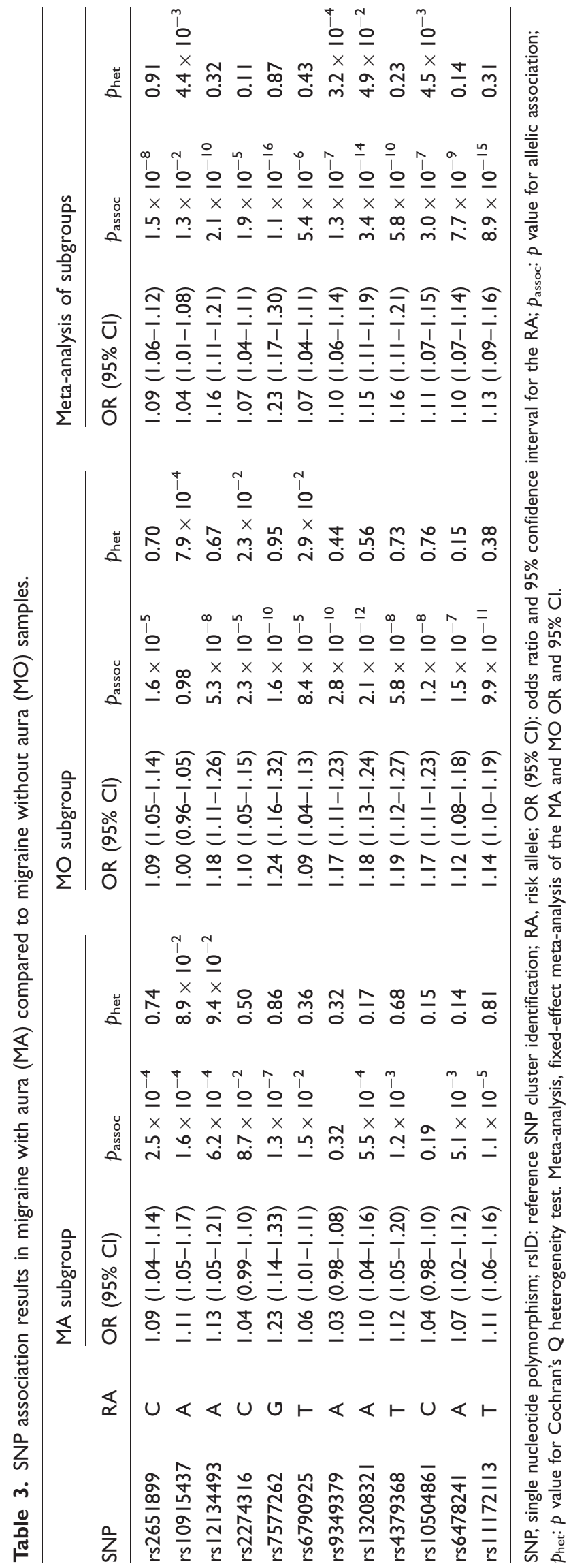

effects for these heterogeneous SNPs were considerably stronger in the MO compared to MA subgroup. Comparing the 12 index SNPs between the clinic- and population-based subgroups (Table 4), we found eight SNPs (rs10915437, rs2274316, rs7577262, rs6790925, rs9349379, rs13208321, rs4379368 and rs6478241) with heterogeneous effects $\left(p_{\text {het }}=8.1 \times 10^{-6}, 3.5 \times 10^{-3}\right.$, $1.1 \times 10^{-2}, \quad 2.0 \times 10^{-5}, \quad 2.6 \times 10^{-2}, \quad 2.0 \times 10^{-2}$, $2.0 \times 10^{-2}$ and $2.7 \times 10^{-5}$, respectively). Although concordant, these eight heterogeneous SNP effects were all considerably larger in the clinic-based subgroup compared to the population-based subgroup. In contrast, the 12 index SNP effects were remarkably homogenous in the female and male subgroups (Table 5). After adjusting for three sets of 12 heterogeneity tests, significant heterogeneity of SNP effects $\left(p_{\text {het }}<1.4 \times 10^{-3}\right)$ remains between the MA and MO subgroups (rs9349379), and clinic- and population-based subgroups (rs10915437, rs6790925 and rs6478241).

Results from SECA (online Supplementary Tables) comparing MA subgroup ORs for the 23,367 index SNPs with the most significant MA association $p$ values $\left(p_{\text {assoc }}\right)$ to the ORs in the MO subgroup indicated the SNP effects were highly concordant across these subgroups. The total number of SNP subsets with nominally significant concordant effects (i.e. Fisher's tests with $\mathrm{OR}_{\mathrm{FT}} \geq 1$ and $p_{\mathrm{FT}} \leq 0.05$ ) was 144 . Out of 1000 replicates, 0 produced 144 subsets with nominally significant concordant effects $\left(p_{\text {FTsig- }}\right.$ permuted $=9.9 \times 10^{-4} ; 95 \%$ CI: $5.1 \times 10^{-5}-5.6 \times 10^{-3}$ ). The subset producing the most significant concordance test was for SNPs with MA $p_{\text {assoc }} \leq 0.3$ and $\mathrm{MO}$ $p_{\text {assoc }} \leq 0.5 \quad\left(\mathrm{OR}_{\mathrm{FT}}=4.4 ; \quad p_{\mathrm{FTmin}}=2.3 \times 10^{-256}\right)$. Analogous concordance was obtained comparing ORs for the 23,162 index SNPs with the most significant MO $p_{\text {assoc }}$ to the ORs in the MA subgroup; here the subset producing the most significant concordance test was for SNPs with MO $p_{\text {assoc }} \leq 0.3$ and MA $p_{\text {assoc }} \leq 0.6$ $\left(\mathrm{OR}_{\mathrm{FT}}=3.8 ; p_{\mathrm{FTmin}}=1.3 \times 10^{-248}\right)$.

Given that the 2013 IHGC MA and MO subgroup analyses utilised overlapping controls, the MA and MO SNP effect estimates are not independent of each other. Furthermore, because $64,847(87 \%)$ of the total 74,239 MA controls were also MO controls, and $93 \%$ of the total 69,427 MO controls were also MA controls, the SECA concordance analysis will be severely biased in favour of concordance - driven by the near identical control allele frequencies being compared to the less similar MA and MO case frequencies. Therefore, SECA was re-run using the independent clinic-based MA (17) and clinic-based MO (18) GWA datasets from the 2013 study (5).

Results from SECA comparing the clinic-based MA subgroup (2849 cases versus 9392 controls, $N_{\text {eff }}=8744$ ) ORs for the 23,182 index SNPs with the most 

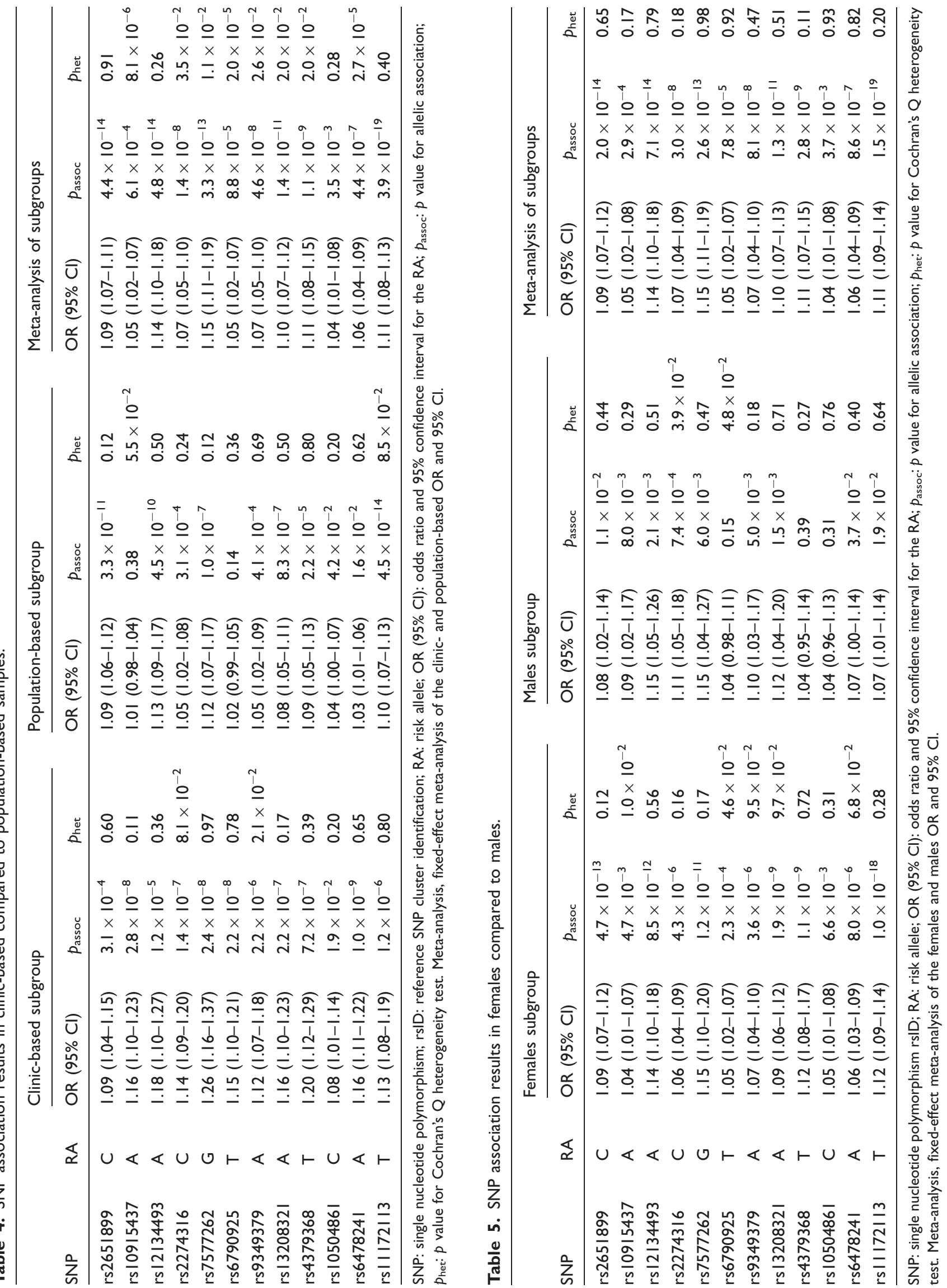
Table 6. SNP effect magnitude comparison for concordant effects.

\begin{tabular}{lllll}
\hline Subgroup I & Subgroup 2 & Total & $\mathrm{OR}_{1}>\mathrm{OR}_{2}$ & $P_{\mathrm{BT}}$ \\
\hline Clinic-based MO & Clinic-based MA & 12,541 & $6780(0.54)$ & $9.4 \times 10^{-20}$ \\
Clinic-based & Population-based & 12,174 & $8529(0.70)$ & $4.9 \times 10^{-324}$ \\
Males & Females & 12,564 & $9420(0.75)$ & $4.9 \times 10^{-324}$ \\
\hline
\end{tabular}

SNP: single nucleotide polymorphism; Total: total number of SNPs with concordant effect across Subgroup I and Subgroup 2; $O R_{1}>O R_{2}$, number of SNPs with odds ratio (OR) for Subgroup I greater than OR for Subgroup 2 (as a proportion of Total is also provided in brackets); $p_{\mathrm{B} T}$ : two-tailed $p$ value from an exact binomial test of observing the number of SNPs with $O R_{1}>O R_{2}$ by chance.

significant clinic-based MA $p_{\text {assoc }}$ to the ORs in the independent clinic-based MO subgroup (2326 cases versus 4580 controls, $N_{\text {eff }}=6170$ ) indicated the SNP effects were highly concordant across these subgroups. The total number of SNP subsets with nominally significant concordant effects was $144\left(p_{\text {FTsig-permuted }}=\right.$ $9.9 \times 10^{-4} ; \quad 95 \% \quad$ CI: $\left.5.1 \times 10^{-5}-5.6 \times 10^{-3}\right)$. The subset producing the most significant concordance test was for SNPs with MA $p_{\text {assoc }} \leq 0.2$ and $\mathrm{MO}$ $p_{\text {assoc }} \leq 0.5 \quad\left(\mathrm{OR}_{\mathrm{FT}}=2.3 ; \quad p_{\mathrm{FTmin}}=1.7 \times 10^{-75}\right)$. Analogous concordance was obtained comparing ORs for the 23,366 index SNPs with the most significant clinic-based MO $p_{\text {assoc }}$ to the ORs in the clinic-based MA subgroup; here the subset producing the most significant concordance test was for SNPs with MO $p_{\text {assoc }} \leq 0.3 \quad$ and $\quad$ MA $\quad p_{\text {assoc }} \leq 0.7 \quad\left(\mathrm{OR}_{\mathrm{FT}}=1.9\right.$; $p_{\text {FTmin }}=2.8 \times 10^{-68}$ ).

Results from SECA comparing ORs for the 23,392 index SNPs with the most significant clinic-based $p_{\text {assoc }}$ to the ORs in the population-based subgroup indicated the SNP effects were highly concordant across these subgroups. All 144 SNP subsets produced nominally significant concordance $\left(p_{\text {FTsig-permuted }}=9.9 \times 10^{-4}\right.$; $\left.95 \% \mathrm{CI}: 5.1 \times 10^{-5}-5.6 \times 10^{-3}\right)$. The subset producing the most significant concordance test was for SNPs with clinic-based $p_{\text {assoc }} \leq 0.1$ and population-based $p_{\text {assoc }} \leq 0.4 \quad\left(\mathrm{OR}_{\mathrm{FT}}=1.7 ; \quad p_{\mathrm{FTmin}}=7.1 \times 10^{-21}\right)$. Analogous concordance was obtained comparing ORs for the 23,374 index SNPs with the most significant population-based $p_{\text {assoc }}$ to the ORs in the clinic-based subgroup; here the subset producing the most significant concordance test was for SNPs with population-based $p_{\text {assoc }} \leq 0.2$ and clinic-based $p_{\text {assoc }} \leq 0.2 \quad\left(\mathrm{OR}_{\mathrm{FT}}=1.8\right.$; $p_{\text {FTmin }}=7.0 \times 10^{-19}$ ).

Results from SECA comparing ORs for the 23,276 index SNPs with the most significant female subgroup $p_{\text {assoc }}$ to the ORs in the male subgroup indicated the SNP effects were highly concordant across these subgroups. All 144 SNP subsets produced nominally significant concordance $\left(p_{\text {FTsig-permuted }}=9.9 \times 10^{-4} ; 95 \%\right.$
CI: $\left.5.1 \times 10^{-5}-5.6 \times 10^{-3}\right)$. The subset producing the most significant concordance test was for SNPs with female $p_{\text {assoc }} \leq 0.3$ and male $p_{\text {assoc }} \leq 0.7\left(\mathrm{OR}_{\mathrm{FT}}=2.0\right.$; $\left.p_{\text {FTmin }}=9.6 \times 10^{-78}\right)$. Analogous concordance was obtained comparing ORs for the 23,266 index SNPs with the most significant male $p_{\text {assoc }}$ to the ORs in the female subgroup; here the subset producing the most significant concordance test was for SNPs with male $p_{\text {assoc }} \leq 0.3 \quad$ and female $\quad p_{\text {assoc }} \leq 0.5 \quad\left(\mathrm{OR}_{\mathrm{FT}}=2.1\right.$; $p_{\text {FTmin }}=1.5 \times 10^{-65}$ ).

Global comparisons of SNP effect magnitude for concordant effects are summarised in Table 6. The random set of independent SNPs encompassed a total of 23,795 SNPs, of which 12,541 SNPs had concordant effects across the clinic-based MA and clinic-based MO subgroups. Of these 12,541 SNPs the effect magnitude was greater in the MO subgroup for $6780(54 \%)$ SNPs (two-tailed binomial test $p_{\mathrm{BT}}=9.4 \times 10^{-20}$ ). Of the 12,174 SNPs with concordant effects across the clinicbased and population-based subgroups, the effect magnitude was greater in the clinic-based subgroup for 8529 $(70 \%)$ SNPs $\left(p_{\mathrm{BT}}<4.9 \times 10^{-324}\right)$. Among the 12,564 SNPs with concordant effects across the female and male subgroups, the effect magnitude was greater in the male subgroup for $9420 \quad(75 \%)$ SNPs $\left(p_{\mathrm{BT}}<4.9 \times 10^{-324}\right)$. Q-Q plots (online Supplementary Figures 1-4) of effect heterogeneity (Cochran's Q) $p$ values between the major migraine subgroups indicate no global heterogeneity between the clinic-based MA and MO (online Supplementary Figure 1), and female and male subgroups (online Supplementary Figure 4) in the total random set of 23,795 independent SNPs, whereas online Supplementary Figure 2 suggests global heterogeneity of effects (represented by an excess of small $p$ values above the null identify line) between the clinic-based and population-based subgroups in the total random set of 23,795 independent SNPs. However, this excess is not present in the random set of 12,174 independent SNPs with concordant effects (online Supplementary Figure 3). 


\section{Discussion}

Recent GWA studies have identified common genetic variants that are associated with migraine (5) and its primary subtypes MA (17) and MO (18). To investigate whether the major migraine subgroups (MA versus MO, clinic- versus population-based, and female versus male) share a common or have distinct genetic aetiologies, we assessed their genetic overlap by examining GWA results from the 2013 IHGC analysis of 23,285 migraine cases and 95,425 population-matched controls of European ancestry.

Although heterogeneity of SNP effects was observed for the 12 genome-wide significant loci between the MA and $\mathrm{MO}$ subgroups, and between the clinic- and population-based subgroups, the risk-increasing allele was the same across all subgroups. This observation extends to the majority of common SNP effects across the human autosome, with SECA finding highly significant evidence that the majority of SNP effects are in the same direction across the MA and MO GWA cases, the clinic- and population-based migraine cases, and the female and male migraine cases. For all of the subgroup comparisons, the proportion of concordant effects increased for SNPs with smaller association $p$ values in one subgroup, and was largest for SNPs with small $p$ values in both subgroups. These findings were not driven by or restricted to the more robust association signals, with the same pattern of SECA results observed after excluding index SNPs with association $p$ values $\left(p_{1}\right)<0.001$ (data not shown).

Comparing the magnitude of SNP effects concordant across clinic-based MA and clinic-based MO subgroups demonstrate the effect magnitudes to be generally larger in the MO subgroup (Table 6). This result was unexpected, given that MA has been shown to have a higher heritability and sibling recurrence risk than MO (3.8 versus 1.9) and is therefore typically considered to be 'more genetic' (i.e. have a higher genetic loading) (19). The significant concordance of common SNP effects across the MA and MO subgroups suggests this observed difference is not due to MA being mediated more by rare variants with larger effect sizes compared to MO. Rather, the difference may be due to a higher degree of heterogeneity among the MA cases, where, for example, genetically distinct subgroups share differing proportions of genetic risk with MO cases.

Contrasting the magnitude of SNP effects concordant across clinic-based and population-based subgroups confirms the effect magnitudes to be generally larger in the clinic-based subgroup (Table 6). This result extends the original study's (5) observation for the 12 genome-wide significant loci and indicates a higher genetic homogeneity and/or loading for the clinic-based samples. This finding is perhaps not surprising given the stricter diagnostic criteria (e.g. compared to self-report) and a higher likelihood that more severe migraine cases (often with a strong family history) would be referred for clinic-based care.

Interestingly, comparing the magnitude of SNP effects concordant across female and male subgroups demonstrates the effect magnitudes to be generally larger in the male subgroup (Table 6). Despite using heterogeneous migraine definitions, the vast majority of twin studies report correlations for migraine in opposite-sex twin pairs to be less than or in between same-sex male and female twin pairs, and several twin studies estimate larger twin correlations for migraine in male-male compared to female-female twin pairs (20). Similar to the twin correlations, our finding of larger SNP effects for male cases compared to female cases suggests migraine may be more genetic, or genetically homogenous, in males compared to females.

Our primary finding of significant SNP effect concordance across these major migraine subgroups indicates fixed-effect meta-analysis of additional GWA datasets, which assumes the same effect size between studies, should continue to identify new SNP loci. For the minority of SNPs with substantial heterogeneity of effect size estimates, alternate meta-analysis models that assume no heterogeneity under the null hypothesis (21), in contrast to the traditional (low-powered) randomeffects model (22), will provide improved power to identify new genome-wide significant loci. Other methods, such as the 'binary-effects model' - a weighted sum of $z$-scores method (23) assigning a greater weight to the studies predicted to have an effect and a smaller weight to the studies predicted to not have an effect (24) - may also improve power to identify new migraine risk loci. Investigation of other migraine subgroups, such as those with and without depression, may also help identify novel risk loci (25).

In summary, these results show the concordant genetic risk across these major migraine subgroups is not restricted to the genome-wide significant/suggestive SNPs identified from the 2013 IHGC fixed-effect meta-analysis of all GWA samples (5). Although not precluding their existence, significantly heterogeneous risk factors across these subgroups (especially with opposite directions) will be the exception rather than the rule. In other words, any differences in common genetic risk across these subgroups is far outweighed by the similarities. Hence, meta-analysis of additional GWA datasets, regardless of their major subgroup composition, will identify new susceptibility loci for migraine. 


\section{Clinical implications}

- For the 12 common migraine risk (single-nucleotide polymorphism (SNP)) loci, the risk-increasing allele was the same across the migraine with aura (MA) and migraine without aura (MO) genome-wide association (GWA) cases, the clinic- and population-based migraine cases, and the female and male migraine cases.

- This observation extends to the majority of common SNP effects (odds ratios) across the human autosome.

- Any differences in common genetic risk across these migraine subgroups are outweighed by the similarities.

- Meta-analysis of additional migraine GWA datasets, regardless of their major subgroup composition, will identify new susceptibility loci for migraine.

\section{Funding}

This research received no specific grant from any funding agency in the public, commercial, or not-for-profit sectors.

\section{Conflict of interest}

None declared.

\section{Acknowledgments}

Dale R Nyholt was supported by the Australian Research Council (ARC) Future Fellowship (FT0991022) and National Health and Medical Research Council (NHMRC) Fellowship (APP0613674) Schemes, by a grant from the NHMRC (APP1075175), and by the European Union's Seventh Framework programme (2007-2013) under grant agreement no. 602633 (EUROHEADPAIN). See the original publication of the 2013 IHGC migraine GWA meta-analysis (5) for detailed cohort-specific acknowledgments.

\section{References}

1. Ligthart L, Boomsma DI, Martin NG, et al. Migraine with aura and migraine without aura are not distinct entities: Further evidence from a large Dutch population study. Twin Res Hum Genet 2006; 9: 54-63.

2. Nyholt DR, Gillespie NA, Heath AC, et al. Latent class analysis does not support migraine with aura and migraine without aura as separate entities. Genet Epidemiol 2004; 26: $231-244$.

3. Russell MB, Rasmussen BK, Fenger K, et al. Migraine without aura and migraine with aura are distinct clinical entities: A study of four hundred and eighty-four male and female migraineurs from the general population. Cephalalgia 1996; 16: 239-245.

4. Russell MB, Ulrich V, Gervil M, et al. Migraine without aura and migraine with aura are distinct disorders. A population-based twin survey. Headache 2002; 42: 332-336.

5. Anttila V, Winsvold BS, Gormley P, et al. Genome-wide meta-analysis identifies new susceptibility loci for migraine. Nat Genet 2013; 45: 912-917.

6. Nyholt DR. SECA: SNP effect concordance analysis using genome-wide association summary results. Bioinformatics 2014; 30: 2086-2088.

7. Willer CJ, Li Y and Abecasis GR. METAL: Fast and efficient meta-analysis of genomewide association scans. Bioinformatics 2010; 26: 2190-2191.
8. The International HapMap Consortium. A second generation human haplotype map of over 3.1 million SNPs. Nature 2007; 449: 851-861.

9. Howie BN, Donnelly P and Marchini J. A flexible and accurate genotype imputation method for the next generation of genome-wide association studies. PLoS Genet 2009; 5: e1000529.

10. Li Y, Willer CJ, Ding J, et al. MaCH: Using sequence and genotype data to estimate haplotypes and unobserved genotypes. Genet Epidemiol 2010; 34: 816-834.

11. Magi R and Morris AP. GWAMA: Software for genomewide association meta-analysis. BMC Bioinformatics 2010; 11: 288

12. Yang J, Weedon MN, Purcell S, et al. Genomic inflation factors under polygenic inheritance. Eur J Hum Genet 2011; 19: 807-812.

13. Ioannidis JP, Thomas G and Daly MJ. Validating, augmenting and refining genome-wide association signals. Nat Rev Genet 2009; 10: 318-329.

14. Zeggini E and Ioannidis JP. Meta-analysis in genomewide association studies. Pharmacogenomics 2009; 10: 191-201.

15. Purcell S, Neale B, Todd-Brown K, et al. PLINK: A tool set for whole-genome association and population-based linkage analysis. Am J Hum Genet 2007; 81: 559-575.

16. R Development Core Team. R: A language and environment for statistical computing. $\mathrm{R}$ Foundation for Statistical Computing, Vienna, Austria, 2009.

17. Anttila V, Stefansson H, Kallela M, et al. Genome-wide association study of migraine implicates a common susceptibility variant on 8q22.1. Nat Genet 2010; 42: 869-873.

18. Freilinger T, Anttila V, de Vries B, et al. Genome-wide association analysis identifies susceptibility loci for migraine without aura. Nat Genet 2012; 44: 777-782.

19. Russell MB and Olesen J. Increased familial risk and evidence of a genetic factor in migraine. BMJ 1995; 311 : 541-544.

20. Mulder EJ, Van Baal C, Gaist D, et al. Genetic and environmental influences on migraine: A twin study across six countries. Twin Res 2003; 6: 422-431.

21. Han B and Eskin E. Random-effects model aimed at discovering associations in meta-analysis of genomewide association studies. Am J Hum Genet 2011; 88: 586-598.

22. DerSimonian R and Laird N. Meta-analysis in clinical trials. Control Clin Trials 1986; 7: 177-188. 
23. de Bakker PI, Ferreira MA, Jia X, et al. Practical aspects of imputation-driven meta-analysis of genome-wide association studies. Hum Mol Genet 2008; 17: R122-R128.

24. Han B and Eskin E. Interpreting meta-analyses of genome-wide association studies. PLoS Genet 2012; 8: e1002555.
25. Ligthart L, Hottenga JJ, Lewis CM, et al. Genetic risk score analysis indicates migraine with and without comorbid depression are genetically different disorders. Hum Genet 2014; 133: 173-186. 